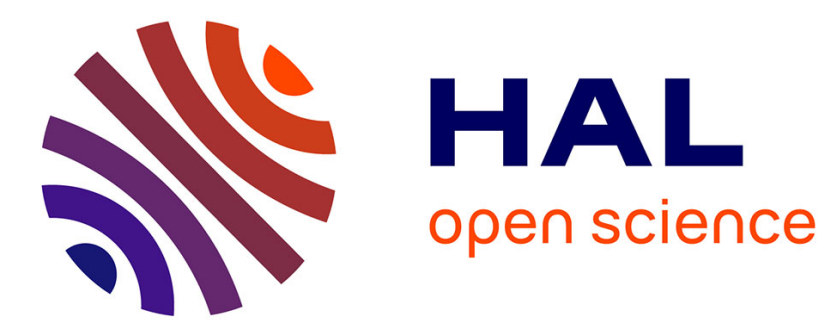

\title{
Le secteur du nettoyage face aux nouvelles technologies
}

Faridah Djellal

\section{To cite this version:}

Faridah Djellal. Le secteur du nettoyage face aux nouvelles technologies. Formation Emploi. Revue française de sciences sociales, 2002, 77, pp.37 à 47 . hal-01085810

\section{HAL Id: hal-01085810 https://hal.science/hal-01085810}

Submitted on 21 Nov 2014

HAL is a multi-disciplinary open access archive for the deposit and dissemination of scientific research documents, whether they are published or not. The documents may come from teaching and research institutions in France or abroad, or from public or private research centers.
L'archive ouverte pluridisciplinaire HAL, est destinée au dépôt et à la diffusion de documents scientifiques de niveau recherche, publiés ou non, émanant des établissements d'enseignement et de recherche français ou étrangers, des laboratoires publics ou privés. 


\title{
Sect eur
d'act ivité \\ Le secteur du nettoyage face aux nouvelles technologies
}

\author{
par Faridah Djellal
}

\begin{abstract}
Le nettoyage, secteur en pleine mutation, développe aujourd'hui des innovations. Le nettoyage des systèmes informatiques, de même que l'informatisation des procédures de nettoyage, bouleversent l'offre des prestations. Différentes trajectoires d'innovation apparaissent, avec chacune leur structure d'emploi. Le secteur se professionnalise, et sa main-d'œuvre se qualifie.
\end{abstract}

Le nettoyage est une activité de service qui connaît aujourd'hui de profondes évolutions : un élargissement et une montée en complexité de l'offre de prestations, une utilisation croissante des technologies, notamment des nouvelles technologies de l'information et de la communication ${ }^{1}$ (NTIC). Ces évolutions ont des incidences importantes sur la structure des emplois de ce secteur, qu'il s'agit d'examiner. Il emploie en France environ 300000 personnes en majorité salariées $(96 \%) .56 \%$ travaillent à temps partiel et $63 \%$ sont des femmes. Il représente environ 11000 entreprises et un pôle artisanal de 6000

\footnotetext{
${ }^{1}$ L'essentiel de nos résultats est issu de l'analyse d'entretiens qualitatifs menés auprès de responsables d'entreprises (directeurs généraux, responsables de départements marketing, informatique et technique) de tailles différentes (grandes entreprises, PME, entreprises familiales). Des entretiens ont également été réalisés auprès des organismes professionnels suivants : la Fédération des entreprises de propreté (FEP), le Centre technique international de la propreté (CTIP), le Fonds d'assurance formation (FAF) (organisme paritaire collecteur agréé des entreprises de propreté), le Comité Liaison Service du Medef - Mouvement des entreprises de France, anciennement CNPF - et enfin le Comité services opérationnels de l'AFAQ (Association française pour l'assurance de la qualité).
}

entrepreneurs inscrits au registre du commerce. Les PME de moins de neuf salariés constituent l'essentiel du secteur (env. 70\%).

Dans une première partie, nous recenserons les principaux domaines d'innovation du secteur : qu'il s'agisse de nouveaux systèmes techniques ou de nouveaux types de prestations. Pour pouvoir rendre compte de manière plus systématique de la dynamique d'innovation à l'œuvre, nous l'aborderons sous l'angle d'une représentation fonctionnelle du produit (Gadrey, 1991), qui décompose toute presta-

Faridah Diellal est maître de conférences en économie à l'université de Lille 1 . Ele est affectée au Centre lillois d'études et de recherche sociologiques et économiques (CLERSE). Ses principaux thèmes de recherche sont l'innovation, la recherche-développement, l'emploi et les qualifications dans les services. 
tion de services en différents types d'opérations de traitement : opérations de traitement de la matière, opérations de traitement de l'information, opérations de services en contact. Nous mettrons ainsi en évidence de multiples trajectoires d'innovation dans le secteur du nettoyage.

La seconde partie vise à mettre en correspondance ces trajectoires d'innovation avec la structure d'emploi de l'activité de nettoyage. Plus précisément, il s'agit d'examiner les conséquences de la modification de la nature du produit sur la nature de « l'emploi », et notamment sur l'émergence de nouvelles modalités de travail liées à la professionnalisation d'une partie non négligeable de cette activité.

\section{L'INNOVATION DANS LE SECTEUR DU NETTOYAGE}

Comme le soulignent Gallouj et Gallouj (1996) la plupart des travaux relatifs à l'innovation dans les services mettent l'accent sur les innovations technologiques. Ils négligent toute autre forme d'innovation. La plupart de ces travaux sont par ailleurs consacrés à ce que Barras (1990) appelle des « services préindustriels ». Il s'agit de services dont le support principal est l'information (banques, assurances, services administratifs, etc.) auxquels on pourrait ajouter la grande distribution. Comme de nombreux autres services opérationnels, le service du nettoyage, dernier refuge des emplois les plus déqualifiés, n'était pas jugé digne d'intérêt. Comment une telle activité aurait-elle pu faire preuve d'une aptitude à l'innovation là où l'ensemble du secteur tertiaire (et en particulier les services intellectuels) s'avérait particulièrement déficient?

Il faut aujourd'hui réviser ce jugement. En effet, avec l'introduction de différents types d'innovations technologiques, mais aussi avec la conception et la fourniture de nouvelles prestations dont certaines marquent une élévation du degré de complexité, le secteur du nettoyage se transforme radicalement. Ces deux aspects de l'innovation seront présentés séparément avant d'être articulés dans une analyse plus systématique à partir d'une décomposition fonctionnelle du produit.

\section{Un recours accru à la technologie et aux NTIC}

Ces dernières années, l'une des évolutions les plus marquantes du nettoyage est la montée en puissance des technologies dans le processus de production. Ces technologies sont à l'origine d'un changement radical de la nature de cette activité longtemps considérée comme essentiellement manuelle.

L'activité de nettoyage a ainsi investi les principaux domaines suivants d'innovations technologiques : la robotique, l'outillage et les petits équipements, les technologies de l'information.

Dans les départements de recherche-développement $(\mathrm{R} \& \mathrm{D})$ de certaines grandes entreprises de nettoyage, la conception, le développement et la production de robots et d'automates sont un phénomène récent. Ces robots ont été conçus pour se substituer au travail humain et améliorer fortement la productivité. Les robots créés ces dernières années par les deux plus grandes entreprises de nettoyage françaises (Abilis, ONET) constituent des innovations de process qui répondent à une logique de coûts, combinée à une nouvelle logique de qualité.

Le groupe hétérogène d'innovations que nous qualifions «d'outillages et de petits équipements » est stimulé par le contexte socioéconomique et technologique. Ces innovations technologiques, qui constituent des innovations de process, sont nombreuses. On citera par exemple : la conception de nouvelles machines (balayeuse aspirante, autolaveuse, chariots modulables en fonction des surfaces) ; la création de nacelles adaptées à la hauteur élevée des nouveaux immeubles ; la création de chariots spécifiques adaptés à la taille des bureaux des clients et équipés de matériels particuliers ; la mise au point d'un système de pulvérisation d'air pour dépoussiérer les unités centrales d'ordinateurs; la conception du bassoumètre (outil qui permet de quantifier l'empoussièrement).

À l'origine, l'activité de nettoyage est une activité non informationnelle. On observe pourtant aujourd'hui une transformation marquée par un recours accru aux technologies de l'information ; les objectifs poursuivis par les entreprises de nettoyage étant les gains de productivité (notamment une meilleure gestion du temps de travail), l'économie des coûts de 
transaction, un meilleur contrôle du travail des salariés, une gestion et un suivi de la qualité de la prestation. Par exemple la création d'un logiciel de contrôle de qualité des dérives possibles par rapport à l'organisation du travail ou aux prestations réalisées. Il recense l'ensemble des tâches à effectuer et leur attribue une note. On peut aussi évoquer la création d'une base de données recensant tous les types de tâches susceptibles d'être réclamées par un client à l'occasion d'une prestation. Cette base de données, analogue à celle utilisée par les consultants en management lors de leurs missions, et plus précisément dans leur recherche de solutions, est régulièrement enrichie de nouvelles expériences, de nouvelles situations, par la direction technique (ou conjointement par les directions informatique et marketing).

Ces innovations technologiques ne suffisent pas, loin s'en faut, à rendre compte des transformations radicales du secteur du nettoyage. Ce secteur est également marqué par l'apparition de fonctions nouvelles.

\section{La montée en puissance des prestations complexes}

La définition actuelle de l'activité de nettoyage est restrictive. Elle ne couvre pas l'ensemble des prestations fournies par les entreprises du secteur qui sont amenées aujourd'hui à offrir des " produits complexes » et à intervenir dans des environnements autres que celui des bureaux ; par exemple l'industrie automobile, l'industrie agroalimentaire, le milieu hospitalier, le secteur du transport ou de l'aérospatiale.

On peut aussi mentionner le cas de l'informatique où certaines entreprises de propreté, encore rares il est vrai, se sont spécialisées dans des niches telle que le nettoyage des micro-ordinateurs, comme l'illustre l'encadré 1.

$\mathrm{Si}$ cette analyse identifie des nouveaux domaines d'innovations technologiques et non technologiques du secteur du nettoyage, elle peine encore à rendre compte de l'ampleur de la dynamique d'innovation du secteur et de ses conséquences sur l'emploi.

\section{Changement du produit et diversité des trajectoires d'innovation}

Nous utilisons la décomposition fonctionnelle du produit proposée par Gadrey (1991), d'une part pour saisir l'évolution du produit de l'activité de nettoyage et sa montée en complexité, et d'autre part pour pouvoir ainsi rendre compte de la diversité des formes et des trajectoires d'innovation. Toute prestation de service peut être envisagée comme la mise en œuvre (dans des proportions variables dans l'espace et dans le temps) d'un certain nombre de fonctions ou groupes d'opérations affectant différents supports (les objets matériels, l'information, l'individu). Les différentes composantes de la prestation sont aussi les suivantes :

- les opérations de logistique et transformation de la matière qui consistent à « traiter » des objets, c'està-dire à les transporter, transformer, entretenir, réparer, etc. ;

- les opérations de logistique et de traitement de l'information qui consistent à « traiter » de l'information « codifiée », c'est-à-dire à la produire, la saisir, la transporter, etc. ;

- les opérations de service en contact ou relationnelles, celles dont le principal support est le client luimême, et qui consistent en un service direct.

\section{Un produit qui se complexifie}

Conformément à la définition traditionnelle du service (Hill, 1977 ; Gadrey, 1991), le nettoyage consiste à transformer ou à améliorer l'état (de propreté) d'un support donné. Ici, il s'agit d'un bien (un matériel, un local, etc.).

Le nettoyage a longtemps été considéré comme une activité peu innovante, utilisant presque exclusivement une main-d'œuvre non qualifiée. Les opérations réalisées nécessitaient peu de contact, et presque aucun traitement de l'information. Le produit (c'est-à-dire la prestation de service de nettoyage $^{2}$ ) se réduisait à sa seule composante logistique matérielle. Cette composante pouvait à elle seule

\footnotetext{
2 Dans le cas des activités de services, la définition du produit est problématique dans la mesure où le produit est un acte, autrement dit un processus.
} 


\section{Encadré 1 \\ La prestation de nettoyage informatique (issu de nos entretiens)}

Cette entreprise de nettoyage a mis en place une innovation qualifiée de " nettoyage informatique ", pour désigner non pas l'usage de l'informatique dans la prestation de nettoyage, mais la prestation de nettoyage des systèmes informatiques. Ce nouveau service porte sur un support inhabituel pour les sociétés de nettoyage (les matériels stratégiques). II a nécessité de multiples modifications qui correspondent à la mise en place d'un nouvel ensemble de caractéristiques et de compétences : recrutement et formation de techniciens aptes à la communication (professionnels de bon niveau bac +2 et +3 ), adaptation des horaires (la prestation a lieu durant les heures ouvrées), mise au point par la direction technique de l'entreprise d'un chariot adapté, ainsi que de produits chimiques spécifiques, de techniques de pulvérisation de l'air et d'aspiration des poussières, méthodologies (protocoles ) de nettoyage, etc.

Ce nouveau service vise à compléter des services que les entreprises de propreté offraient déjà, en particulier dans le secteur tertiaire (banques et assurances). On peut reconstruire le processus de mise au point et de lancement de ce nouveau service dans les termes suivants :

Initialement, le cahier des charges (défini conjointement par le client et l'entreprise de nettoyage) interdisait explicitement aux entreprises de propreté de nettoyer les micro-ordinateurs, probablement parce que les clients estimaient que le personnel de nettoyage n'était pas qualifié, qu'il était incompétent. La direction de l'entreprise de nettoyage réalise une enquête auprès des directions informatiques des firmes clientes sur leur perception de l'entreprise de nettoyage et sur les besoins de nettoyage des matériels informatiques. II en ressort l'existence d'un besoin réel, mais aussi le sentiment de l'inaptitude des firmes de nettoyage à le satisfaire. La direction de l'entreprise de nettoyage propose alors un service de nettoyage informatique avec une équipe spécialisée dans ce domaine, capable de prendre en charge à la fois l'entretien des locaux et celui du matériel informatique, dans une logique de service complet.

Le résultat a été à la fois la construction d'une nouvelle " professionnalité » et celle de nouveaux systèmes techniques. Les employés sont des techniciens qui vont intervenir aux heures de bureaux (contrairement au nettoyage traditionnel). Ils ont des tenues de travail spécifiques ; ils savent communiquer et disposent d'un petit chariot spécifique (avec des produits souvent fabriqués par la direction technique). Ils ouvrent les claviers, les unités centrales et nettoient les différents éléments selon une méthodologie établie (protocole).

II a fallu résoudre de nombreux problèmes techniques : adapter la taille des chariots à l'exiguïté des bureaux ; réfléchir à la manière de dépoussiérer l'unité centrale - constituée de composants électroniques fragiles -, sans endommager le matériel. La direction a ainsi mis au point un système de pulvérisation d'air (un petit système d'aspirateur avec une soufflette), suffisamment puissant pour bouger la poussière et la collecter en même temps. 
définir le produit de l'activité de nettoyage. Ce jugement reste valable pour les petites entreprises (souvent familiales) offrant uniquement des prestations simples (le nettoyage courant des bureaux par exemple).

Le produit (ou prestation) des entreprises de grande taille, quant à lui, ne peut plus être envisagé selon cette seule composante matérielle. Dans une perspective dynamique, on peut rendre compte de l'évolution des prestations par l'examen des opérations réalisées par les entreprises de nettoyage.

Le produit de l'activité de nettoyage se décompose aujourd'hui pour l'essentiel en trois types d'opérations :

1) Des opérations de dans la mesure où le support dominant est toujours un bien (nettoyage d'un matériel, d'un local, etc.). Cependant, il est important de souligner que les techniques mobilisées sont différentes selon les entreprises. En effet, certaines d'entres elles utilisent toujours des techniques manuelles (balai et serpillière) tandis que d'autres sont à l'origine d'innovations technologiques plus ou moins sophistiquées ou s'appuient sur elles. Cette distinction selon la technique utilisée (technologie matérielle simple ou technologie matérielle complexe) est essentielle pour saisir la dynamique d'innovation des entreprises de nettoyage et les conséquences sur leur structure d'emploi.

2) Des opérations de logistique de traitement de l'information. Ainsi, l'activité de nettoyage investit le domaine des technologies de l'information à la fois pour des usages internes et externes (c'est-à-dire pour le client et l'entreprise de nettoyage) avec des objectifs de gestion des temps de travail, de gestion de la qualité, d'évaluation de la performance, etc. Les principaux outils développés sont des bases de données, des outils de contrôle de qualité, etc.
3) Des opérations de service en contact ou relationnelles. Dans ce cas, le support dominant du service est le client. Il s'agit ici d'une diversification et d'une spécialisation de l'offre des entreprises de propreté. Par exemple, l'une d'entre elle a développé pour l'aéroport de Roissy, où elle intervenait déjà dans le cadre de prestations traditionnelles de nettoyage, un service d'assistance aux personnes blessées et rapatriées. On peut aussi évoquer le développement des services d'accueil dans le secteur de l'hôtellerie (les chaînes d'hôtels type « Formule 1 » par exemple, au sein desquelles les entreprises de nettoyage interviennent déjà dans le cadre de prestations traditionnelles).

Ces deux derniers types d'opérations (traitement de l'information et opérations relationnelles) constituent les deux grandes transformations radicales de l'activité de nettoyage, encore considérée à tort comme une activité manuelle. L'émergence de ces nouvelles opérations, la coexistence (ou combinaison) de certaines d'entres elles, voire des trois (en incluant les opérations de logistique matérielles), conduisent à l'apparition de différentes trajectoires d'innovation dans les entreprises de nettoyage

\section{De multiples trajectoires d'innovation}

Ainsi, la trajectoire d'innovation ${ }^{3}$ des entreprises de nettoyage ne repose pas seulement sur les technologies « matérielles » (c'est-à-dire associées à la composante logistique matérielle du produit) qu'elles soient simples ou complexes. Il y a un recours accru aux technologies de l'information. Par ailleurs, la technologie ne suffit pas à elle seule à rendre compte de l'ensemble des phénomènes d'innovation.

Ainsi la classification de Pavitt (1984), qui décrit l'ensemble des services par une trajectoire technologique qualifiée de «dominée par les fournisseurs » (c'est-à-dire de simple adoption par les services de systèmes techniques produits ailleurs) ne rend pas compte de ces nouveaux phénomènes, et plus particulièrement des diverses formes et des trajectoires d'innovation à l'œuvre dans une même entreprise ou au sein des différentes entreprises d'un même secteur.

\footnotetext{
${ }^{3} \mathrm{Au}$ sens de la théorie évolutionniste du changement technique
} (Dosi, 1982). 
Gallouj (1999) propose une réflexion sur l'enrichissement de ces trajectoires pour l'ensemble des services. Sur la base de la décomposition fonctionnelle de l'activité de service, cet auteur met en évidence cinq trajectoires d'innovation :

$\rightarrow$ une trajectoire de logistique et de transformation matérielle qui affecte la composante « logistique matérielle » de la prestation de service et qui traduit la dynamique des technologies de transport et traitement de la matière (véhicules, système de cuisson et de réfrigération, par exemple);

$\rightarrow$ une trajectoire de logistique et de traitement de l'information, qui affecte la composante « traitement de l'information » de la prestation, et qui traduit l'évolution des technologies de l'information et de communication ;

$\rightarrow$ une trajectoire méthodologique ou cognitive (importante dans les activités de conseil) qui rend compte de l'évolution des méthodologies de traitement de la connaissance ;

$\rightarrow$ une trajectoire servicielle (pure) que Gallouj (1999) considère comme un idéal-type décrivant l'innovation de service indépendamment de tout moyen technique, c'est-à-dire par «mobilisation directe de compétences pour fournir les fonctions ou les caractéristiques de services ». On peut citer l'exemple de la prise en charge de champs d'expertise nouveaux dans le conseil.

$\rightarrow$ une trajectoire relationnelle qui affecte la composante « relationnelle ou « service en contact» de la prestation, et qui décrit en particulier l'apparition et l'évolution de nouvelles modalités de mise en relation du client et du prestataire.

Ces différentes trajectoires peuvent être à l'œuvre dans une même activité de service. Certaines d'entre elles peuvent être plus importantes que d'autres et différentes combinaisons possibles de trajectoires peuvent être envisagées.

On retrouve ces différentes trajectoires d'innovation dans les entreprises de nettoyage. Il faut cependant établir une distinction entre les petites et les grandes entreprises. L'activité des petites entreprises de nettoyage se réduit à la composante logistique matérielle du produit et mobilise des techniques matérielles simples (conformément à la distinction établie précédemment). Il s'agit souvent d'entreprises familiales qui n'innovent pas et qui n'ont pas encore investi (ou peu) le domaine des technologies de l'information. Dans ce cas, la trajectoire d'innovation est limitée.

Quant aux entreprises de grande taille, nous avons montré que leur produit combinait de manière significative les cinq composantes (matérielle, informationnelle, servicielle, relationnelle et méthodologique).

De nombreux exemples d'innovations rendent compte des évolutions de ces différentes trajectoires au sein des grandes firmes de nettoyage.

Les trajectoires servicielles et relationnelles semblent difficiles à dissocier. On peut les illustrer par la multiplication de nouveaux services comme par exemple :

- Le bionettoyage hospitalier ${ }^{4}$. Ce nouveau type de prestations a nécessité un élargissement des compétences et par conséquent une professionnalisation du secteur. Le personnel de certaines entreprises de nettoyage s'est substitué à celui de l'hôpital (notamment au personnel aide-soignant) pour le nettoyage des blocs opératoires. Dans ce même milieu hospitalier, certaines entreprises de nettoyage, parfois partenaires de sociétés de restauration collective, ont développé un service qui consiste à apporter un soin particulier à la distribution et à la manipulation des plateaux repas.

- le nettoyage intégré au processus de production et aux contrôles bactériologiques dans l'industrie agroalimentaire.

La trajectoire logistique matérielle complexe peut être mise en évidence par la robotisation de certains processus de nettoyage, la mise en place de nacelles spécifiques ou de chariots adaptés.

- La trajectoire logistique informationnelle, plus récente dans les firmes de nettoyage, peut être illustrée par la conception et l'utilisation de nombreux systèmes logiciels, par exemple de contrôle de la qualité, de définition des tâches, etc.

\footnotetext{
${ }^{4}$ Il s'agit de l'adaptation de méthodes et techniques de nettoyage aux exigences de l'hygiène dans les milieux hospitaliers. Soulignons que le bionettoyage constitue un atout dans la prévention des infections nosocomiales (maladies que l'on contracte à l'hôpital) (cf. Économie et Géographie, n 307,1993).
} 
- La trajectoire cognitive ou méthodologique est sans doute une trajectoire plus faible pour l'instant. Elle se traduit par exemple par la création de sociétés de conseils en nettoyage et la mise en place de méthodologies ou protocoles de nettoyage.

Ainsi, le produit de l'activité de nettoyage se complexifie. Il se traduit par un enrichissement des opérations réalisées, sous l'impulsion de différentes variables qualitatives donnant naissance à diverses trajectoires d'innovation. Quelles sont les incidences de l'enrichissement du produit de l'activité de nettoyage sur l'emploi et sur l'organisation du travail ?

\section{TRAECTOIRES D'IN NOVATION ET EMPLOI}

Il n'est pas évident, a priori, d'étudier l'impact de la transformation de l'activité de nettoyage sur l'emploi. Cela est particulièrement vrai quand on se limite à un inventaire statique de la structure d'emploi. Si on s'intéresse plus systématiquement à la nature du système d'emploi du secteur du nettoyage, on met en évidence la coexistence de deux modèles d'organisation du travail qui s'articulent avec les différentes trajectoires d'innovation envisagées précédemment.

\section{La structure d'emploi du secteur du nettoyage}

L'emploi dans le secteur du nettoyage présente un certain nombre de caractéristiques :

- L'activité de nettoyage n'échappe pas à la règle de la surreprésentation de l'emploi féminin dans la plupart des activités tertiaires (Gadrey, 1997). On observe un taux élevé de féminisation de l'emploi salarié (75\% en 1995). Il s'agit de l'un des taux les plus élevés parmi les services.
- Cette activité a massivement recours à une maind'œuvre pas ou peu qualifiée. Les ouvriers qualifiés et non qualifiés représentent l'essentiel de la main-d'œuvre. On observe néanmoins une baisse de cette main-d'œuvre ouvrière (notamment non qualifiée). Cette légère baisse s'explique par les programmes de formation mis en place ces dernières années par l'INHNI ${ }^{5}$ et surtout par les initiatives de certaines grandes entreprises de nettoyage qui forment leurs salariés au sein de leurs centres de formation. Les contextes économique et surtout technologique analysés précédemment (c'est-à-dire l'utilisation massive des nouvelles technologies et la diversification et spécialisation de l'offre) sont les principaux stimulants à la mise en place de ces formations. En effet, il s'agit de former les individus à la réalisation de prestations plus complexes et à l'usage de technologies plus sophistiquées (on passe du balai à l'ordinateur).

- Le taux d'encadrement est l'un des plus faibles. Mais là aussi, on observe une évolution liée principalement à la mise en place récente par le ministère de l'Éducation nationale de formations telles que : le CAP «maintenance et hygiène des locaux » (1987), le BEP « bioservice » (1989), le bac professionnel « hygiène et environnement » (1991), le BTS « hygiène, propreté, environnement », le $\mathrm{bac}+3$ (licence professionnelle de «management, hygiène et services de propreté »). L'arrivée de ces jeunes diplômés dans la profession et les initiatives des centres de formation interne sont en mesure de renforcer le début de professionnalisation et de contribuer à accroître le taux d'encadrement.

- Lié à « l'emploi féminin », le temps partiel est fortement développé dans le nettoyage. En effet, les entreprises de nettoyage interviennent en dehors des horaires de travail de leurs clients. Il est alors possible, notamment pour les ouvriers non qualifiés, de combiner différents contrats. La moyenne hebdomadaire de temps de travail chez ces derniers excède rarement les 32 heures. En 1991, seulement $1,2 \%$ de cette population ouvrière avait une durée journalière de travail supérieure à trois heures.

\footnotetext{
${ }^{5}$ Institut national de l'hygiène et du nettoyage industriel.
} 
- La possibilité de multiplier de nombreux petits contrats dans les entreprises de nettoyage renvoie à la notion de flexibilité quantitative (c'est-à-dire à l'adaptation continue du volume de la maind'œuvre en réponse aux fluctuations de la demande ${ }^{6}$ ) qui représente une donnée structurelle de la gestion de la main-d'œuvre dans ce secteur. Cependant, les contrats multiples s'appliquent principalement aux ouvriers dépourvus de qualification ou aux étrangers en voie d'insertion sociale et/ou professionnelle.

Lensemble de ces caractéristiques représentatives d'un grand nombre d'entreprises de nettoyage ${ }^{7}$ doit être nuancé en raison de la modification de la nature du produit ; cette modification se traduit d'une part, par des innovations de produit, de process, et des innovations sociales (importance accordée aux notions de services et de relation de service) et d'autre part, par l'introduction des technologies de l'information dans le processus de production. Cet ensemble de caractéristiques doit être adapté à l'évolution des trajectoires des entreprises. Nous avons en effet montré que le secteur du nettoyage était marqué par la coexistence de différentes trajectoires d'innovation.

Pour rendre compte des conséquences sur l'emploi de l'émergence de ces différentes trajectoires d'innovation, nous proposons une analyse en termes de système d'emploi ${ }^{8}(c f$. Bertrand, 1991 ; Gadrey, 1991).

\section{Quelles incidences pour l'emploi, la formation et la qualification?}

À l'aide de la décomposition fonctionnelle du produit (à savoir la décomposition de la prestation de service en différentes opérations de traitement : de la matière, de l'information et du service en contact), nous avons montré que l'activité de nettoyage s'est profondément modifiée ces dernières années. On peut résumer les principales caractéristiques actuelles du système d'emploi dans les entreprises de nettoyage en partant des facteurs socio-économiques et technologiques.

\footnotetext{
${ }^{6}$ Définition de Michon F. (1987) reprise dans Gadrey J. et N. (1991).

${ }^{7}$ Rappelons que les PME de moins de neuf salariés représentent l'essentiel du secteur (à peu près $70 \%$ ).

${ }^{8}$ Ce qui revient à analyser les formes d'emploi, la structure des qualifications et les salaires. La question des rémunérations n'est pas traitée dans cet article.
}

Les résultats présentés dans le tableau 1 reprennent bien sûr les caractéristiques traditionnelles relatives à une partie de l'activité de nettoyage, mais ils rendent compte aussi de la montée en complexité du produit, qui s'est traduite, rappelons-le, par l'apparition d'opérations de nature informationnelle et de service en contact (ou relationnelle).

Cette analyse en termes de systèmes d'emploi nous amène à nous interroger sur les modalités d'organisation du travail et de gestion des ressources humaines dans le contexte de la montée en complexité du produit de l'activité de nettoyage.

Pour Gadrey (1997 ; 1999), il existe un modèle de flexibilité segmenté de l'emploi dans les services, décomposable en deux modèles, selon les pratiques de gestion des ressources humaines des entreprises. Il y aurait ainsi, selon les cas, un modèle néotaylorien de flexibilité quantitative et un modèle d'adaptabilité organisationnelle. Au sein du premier modèle, les salariés sont directement les victimes des contraintes et des aléas de production. Dans ce modèle prédomine la logique de réduction des coûts. Le second modèle peut se résumer dans les termes suivants : une gestion plus souple des contraintes de production, une implication maximale de la main-d'œuvre et sa fidélisation et un noyau dur auquel appartient la majorité du personnel.

On est amené à faire l'hypothèse de la coexistence de ces deux modèles d'organisation du travail dans le secteur du nettoyage. En effet, si on se limite à une analyse statique de l'activité de nettoyage en reprenant les principales données quantitatives et qualitatives de la structure d'emploi, on est tenté de conclure à la domination du modèle néotaylorien de flexibilité quantitative. Jusqu'à aujourd'hui, ce dernier semble le plus répandu parmi les activités de services, notamment « manuelles » ou peu qualifiées. Les attributs de ce modèle correspondent en effet aux principales caractéristiques (ou attributs) relatives aux entreprises de nettoyage caractérisées par l'existence unique ou la domination de la composante matérielle. Ce modèle s'applique aux entreprises offrant des prestations traditionnelles de base et peu concernées par l'innovation au sens large.

En revanche, si l'on tient compte des innovations développées récemment par les entreprises de nettoyage, de la montée en complexité du produit de cette activité, c'est-à-dire à la fois du contexte socio- 
Tableau 1

\section{Principales transformations du système d'emploi dans les entreprises de nettoyage (inspiré de J. Gadrey, 1990)}

\begin{tabular}{|c|c|c|}
\hline $\begin{array}{l}\text { Transformation des conditions } \\
\text { économiques et techniques de } \\
\text { l'activité }\end{array}$ & $\begin{array}{c}\text { Implication sur la nature } \\
\text { des tâches et les exigences } \\
\text { de qualification }\end{array}$ & $\begin{array}{l}\text { Caractérisation du mode } \\
\text { de gestion de la main- } d \text { 'œuvre }\end{array}$ \\
\hline $\begin{array}{l}\text { Conditions économiques : } \\
\text { - Activité fortement externalisée. } \\
\text { - Passage à une logique de } \\
\text { diversification et de } \\
\text { complexification des } \\
\text { produits' services en fonction } \\
\text { du type de clientèle. } \\
\text { - Passage d'une logique par le } \\
\text { prix à une logique par la qualité } \\
\text { pour les prestations complexes. } \\
\text { - Passage d'une activité ‘nnvisible» } \\
\text { à une activité visible (liée à } \\
\text { l'évolution des mentalités). } \\
\text { - Implication du prestataire dans } \\
\text { le processus de transaction. }\end{array}$ & $\begin{array}{l}\text { - Passage de sans qualification } \\
\text { à une qualification. } \\
\text { - Polyvalence pour les ouvriers } \\
\text { qualifiés. } \\
\text { - Logique de professionnalisation } \\
\text { (apparition de nouveaux métiers } \\
\text { liés au marketing, au contrôle } \\
\text { de qualité, aux méthodes, } \\
\text { au commercial, au conseil). } \\
\text { - Apparition dans certains cas } \\
\text { de structures de recherche- } \\
\text { développement avec donc } \\
\text { de nouvelles compétences, } \\
\text { de nouveaux métiers. } \\
\text { - Coexistence de plusieurs } \\
\text { trajectoires: des trajectoires } \\
\text { servicielle, de logistique } \\
\text { matérielle complexe et de } \\
\text { logistique informationnelle. } \\
\text { Ces différentes trajectoires peu- } \\
\text { vent correspondre à différentes } \\
\text { structures organisationnelles: } \\
\text { Structure simple, adhocratie et } \\
\text { récemment matricielle. }\end{array}$ & $\begin{array}{l}\text { - Efort en matière de formation } \\
\text { interne et externe. } \\
\text { - Enrichissement des tâches. } \\
\text { - Création d'instituts de formation } \\
\text { interne pour les prestations } \\
\text { complexes. } \\
\text { - Création de diplômes publics } \\
\text { (bac professionnel, BTS, } \\
\text { voire bac + } 4 \text { à 5, } \\
\text { diplôme IUP- Institut universtaire } \\
\text { professionnalisé). } \\
\text { - Póle croissant du marché du } \\
\text { travail externe pour les fonctions } \\
\text { professionnelles (informatique, } \\
\text { marketing, etc.) }\end{array}$ \\
\hline $\begin{array}{l}\text { Processus technique : } \\
\text { - Passage de l'utilisation } \\
\text { des technologies logistiques } \\
\text { (traitement de la matière) } \\
\text { à l'utilisation des technologies } \\
\text { informationnelles (traitement } \\
\text { de l'information). } \\
\text { - Procédures de contrôle. } \\
\text { - Automatisation de certaines } \\
\text { machines (apparition des robots). } \\
\text { - Conception moderne des } \\
\text { bâtiments et des machines. }\end{array}$ & $\begin{array}{l}\text { - Baisse des opérations } \\
\text { de logistique matérielle simple } \\
\text { remplacées par des opérations } \\
\text { de logistique matérielle } \\
\text { complexe. } \\
\text { - Apparition des opérations } \\
\text { de traitement de l'information } \\
\text { et de service en contact ou } \\
\text { relationnelle. }\end{array}$ & $\begin{array}{l}\text { - Rexibilité quantitative importante } \\
\text { pour les opérations de logistique } \\
\text { matérielle. } \\
\text { - - Rexibilité quantitative représente } \\
\text { un frein à l'innovation pour } \\
\text { les opérations de logistique } \\
\text { informationnelle et servicielle. }\end{array}$ \\
\hline
\end{tabular}


économique et de la dynamique technologique, on arrive à des résultats dont l'interprétation peut être sensiblement différente de la précédente.

Plusieurs arguments justifient ces résultats divergents. Ils tiennent aux nouvelles opérations (informationnelle, servicielle) qui constituent aujourd'hui une composante de plus en plus essentielle de l'activité de nettoyage :

- Les contraintes d'aménagement de temps de travail liées à celles du client (contraintes très répandues dans les opérations relevant des prestations de base) sont ici plus limitées, voire nulles. On peut supposer que la possibilité de multiplier les contrats pour un même salarié est difficilement applicable, voire néfaste à l'innovation. L'émergence des dimensions servicielle et relationnelle conduit le prestataire à limiter les pratiques de temps partiel. Cette nouvelle limite s'impose au prestataire afin de fidéliser, motiver et impliquer ses salariés. D'après nos entretiens, ces différents comportements ne peuvent s'appliquer à un salarié contraint de travailler pour différentes entreprises et qui doit faire face à un problème d'identification'. Ce problème d'identification est en contradiction avec les notions de service, de relation de service et donc de qualité de service (c'est-à-dire avec l'apparition des opérations de service en contact ou relationnelle). On peut en conclure que la flexibilité quantitative, notamment externe, sera beaucoup plus faible. $\mathrm{La}$ pratique de celle-ci peut être interprétée comme un frein important à la réalisation des opérations de service en contact.

- L'introduction récente des technologies de l'information a aussi pour conséquence l'apparition de nouvelles fonctions. On peut citer bien sûr le cas des fonctions informatique, marketing, méthodes et R\&D. Ces nouvelles fonctions donnent le jour à de nouveaux métiers (commerciaux, informaticiens, formateurs, contrôleurs de qualité, etc.) inexistants à l'origine dans ce type d'activité. On peut aussi souligner l'apparition de nouvelles « tâches » plus complexes, comme la formalisation des procédures internes de réalisation de la prestation, la formalisa-

\footnotetext{
${ }^{9} \mathrm{Il}$ arrive fréquemment qu'un employé d'une entreprise de nettoyage s'identifie à l'entreprise-cliente. Il reconnaît plus volontiers travailler pour cette dernière que pour l'entreprise de nettoyage : «c'est plus prestigieux. »
}

tion des procédures des relations avec les clients, etc. On peut également citer l'apparition de nouveaux métiers à contenu relationnel (commerciaux, formateurs, contrôleurs de qualité, etc.) (Djellal, 1998).

- Ces nouvelles fonctions, ces nouveaux métiers liés aux opérations de logistique informationnelle, servicielle (et relationnelle) témoignent d'une montée en professionnalisation du secteur du nettoyage.

- La réalisation de ces nouvelles opérations (informationnelle et servicielle) contribue d'une certaine façon à diminuer le taux des ouvriers non qualifiés au profit des ouvriers qualifiés. Dans le même temps, les innovations de produit conduisent à l'apparition de nouvelles fonctions citées précédemment qui ont pour conséquence une augmentation des ETAM (employés, techniciens, agents de mâ̂trise) et surtout des cadres.

Le contexte économique et technologique a contribué à l'élévation du niveau de formation ou de qualification des ouvriers qualifiés et des ETAM. L'apparition d'un certain nombre de diplômes nationaux évoqués précédemment, et de programmes de formation mis en place par les entreprises de nettoyage, ont contribué à cette élévation du niveau. Les différents types de formation par qualification sont illustrés dans le tableau 2.

L'analyse de la transformation du système d'emploi dans le nettoyage s'est appuyée principalement sur la complexification du produit de cette activité. Nous avons notamment souligné que la montée en complexité du produit était marquée par l'apparition d'opérations de traitement de l'information et d'opérations de service ou relationnelle dont les caractéristiques, par rapport à l'emploi, sont différentes de celles du modèle néotaylorien de flexibilité quantitative.

Le nouveau modèle en émergence dans le secteur du nettoyage peut également être qualifié de «modèle d'adaptabilité organisationnel » comme l'a défini J. Gadrey. Ce modèle rend bien compte des transformations à l'œuvre dans cette activité. Il traduit un changement organisationnel radical dans les services de nettoyage. Les opérations de logistiques informationnelle et servicielle ne peuvent se développer sur le même modèle que les opérations de logistique matérielle simple ; en effet, elles nécessitent de nou- 
Tableau 2

\section{Les types de formations dispensées par qualification selon les objectifs}

\begin{tabular}{|l|l|l|}
\hline \multicolumn{1}{|c|}{ Type de qualification } & \multicolumn{1}{c|}{ Type de formation } & \multicolumn{1}{c|}{ Objectifs } \\
\hline $\begin{array}{l}\text { Ouvriers } \\
\text { (principalement qualifiés). }\end{array}$ & $\begin{array}{l}\rightarrow \text { Formation continue dispensée } \\
\text { au sein des centres de formation } \\
\text { des entreprises de nettoyage. }\end{array}$ & $\begin{array}{l}\text { - Acquérir des compétences } \\
\text { supplémentaires notamment } \\
\text { techniques (utilisation de nouveaux } \\
\text { matériels) } \\
\text { ouvriers polyvalents } \\
\text { ex : le nettoyage du matériel } \\
\text { informatique. }\end{array}$ \\
\hline $\begin{array}{l}\text { Employés, techniciens, } \\
\text { agents de maîtrise. }\end{array}$ & $\begin{array}{l}\rightarrow \text { Formation continue pour } \\
\text { certaines tâches spécifiques } \\
\text { à l'activité. }\end{array}$ & $\begin{array}{l}\text { - Acquérir de nouvelles compétences } \\
\text { de type techniques, mais aussi } \\
\text { relationnelles. } \\
\text { ex : les services d'accueil dans le } \\
\text { secteur de l'hôtellerie ou le rapatrie- } \\
\text { ment des blessés dans les aéroports. } \\
\text {-Qualification spécifique seulement } \\
\text { disponible sur le marché exteme. }\end{array}$ \\
& $\begin{array}{l}\rightarrow \text { Formation initiale (création de } \\
\text { au diplômes nationaux du CAP depuis 1987). }\end{array}$ & \\
\hline
\end{tabular}

velles pratiques de gestion des ressources humaines, des investissements importants en technologies de l'information et en formation (création de plusieurs centres de formation internes aux entreprises de nettoyage ${ }^{10}$ ). Une montée en professionnalisation a aussi renforcé le noyau dur (cadres, spécialistes, employés permanents).

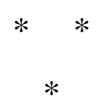

Ainsi, au sein du secteur du nettoyage deux «modèles »d'innovation coexistent : les entreprises de petite taille, à caractère familial, sont enfermées dans une trajectoire de logistique matérielle simple où dominent les techniques manuelles. Les entreprises

\footnotetext{
${ }^{10}$ Certaines entreprises de nettoyage, c'est-à-dire les plus importantes en termes de personnel et de chiffre d'affaires, ont créé leur propre école. L'objectif de ces écoles était de répondre à un « vide fonctionnel ». En effet, en termes de main-d'œuvre, elles avaient des besoins spécifiques qu'elles ne pouvaient pas satisfaire sur le marché du travail.
}

de grande taille ont modifié la nature de leur produit en renforçant leur composante « logistique et transformation matérielle » (introduction de techniques complexes liées à la mécanique, à la robotique). Les étapes suivantes ont consisté en une montée en puissance des dimensions servicielle et relationnelle du produit et des trajectoires d'innovation correspondantes.

Si pour la première catégorie d'entreprises peu de changements s'opèrent dans la nature des tâches, dans les qualifications exigées, il en va tout autrement pour les secondes. En effet, si le modèle néotaylorien de flexibilité quantitative s'applique aux entreprises caractérisées par une trajectoire de logistique matérielle simple, en revanche il ne peut s'appliquer aux entreprises poursuivant d'autres trajectoires d'innovation (logistique informationnelle et relationnelle). Un nouveau modèle émerge à travers ces entreprises - le modèle d'adaptabilité organisationnel - jusqu'à présent à l'œuvre dans les entreprises (souvent informationnelles) utilisant plutôt une main-d'œuvre qualifiée. 


\section{Bibliographie}

Barcet A., Bonamy J., Mayère A. (1987), Modernisation et innovation dans les services aux entreprises, Commissariat général du Plan.

Barras R. (1990), « Interactive Innovation in Financial and Business Services : The Vanguard of the Service Revolution », Research Policy, $\mathrm{n}^{\circ}$ 19, pp. 215-237.

Bertrand O. (1991), « Les systèmes d'emploi tertiaire : différences et convergences », in Gadrey J. et Gadrey N., La gestion des ressources humaines dans les services et le commerce : flexibilité, diversité compétitivité, L'Harmattan, coll. « Pour l'emploi ».

Bertrand O. (1998), «Qualité et hétérogénéité des emplois de services », Formation Emploi, $\mathrm{n}^{\circ} 23$, juillet-septembre.

Bousquet N., Grandgérard C. (1994), Professionnalisation du secteur du nettoyage industriel : Évolution des fonctions et des formations, Programme FORCE.

Djellal F. (1995), Changement Technique et conseil en technologie de l'information, L'Harmattan, Coll. «Logiques Économiques ».

Djellal F. (1998a), « Innovation in the french contract cleaning industry », in Djellal F., Gallouj F. et C.

Innovation trajectories in french service industries, Rapport pour la Commission Européenne, DG XII, Programme TSER, projet SI4S, juillet.

Djellal F., Gallouj F., Gallouj C. (1998), Innovation trajectories in french service industries, Rapport pour la Commission Européenne, DG XII, Programme TSER, projet SI4S, juillet.

Djellal F. (2000), « Technologies de l'information et services non informationnels : les services non informationnel », Économie et Sociétés, série EGS, n² 2 , juin.

Dontenwill E., (1993), Approche qualitative des secteurs de services marchands : le nettoyage industriel,
Institut de Recherche de l'Entreprise, Groupe ESC Lyon.

Dosi G. (1982), «Technological paradigms and technological trajectories, a suggested interpretation of the determinants and directions of technological change », Research Policy, vol. 11.

Gadrey J. (1990), « Les systèmes d'emplois tertiaires au cœur des transformations du marché du travail », Formation Emploi, n 29 , janvier-mars, pp. 21-34.

Gadrey J. (1991), « Le service n'est pas un produit : quelques implications pour l'analyse économique et pour la gestion », Politiques et Management Public, vol. $9, \mathrm{n}^{\circ} 1$.

Gadrey J., Gadrey N. (ed.) (1991), La gestion des ressources humaines dans les services et le commerce: flexibilité, diversité compétitivité, L'Harmattan, coll. «Pour l'emploi ».

Gadrey J. (1997), La professionnalisation des emplois tertiaires à prédominance féminine, Conférence de l'OCDE, 11 et 12 décembre, Paris.

Gallouj F. (1991), « Les formes de l'innovation dans le conseil », Revue d'Économie Industrielle, $\mathrm{n}^{\circ} 57$.

Gallouj C., Gallouj F. (1996), L'innovation dans les services, Économica.

Gallouj F. (1999), « Les trajectoires de l'innovation dans les services : vers un enrichissement des taxonomies évolutionnistes », Économies et Sociétés, série EGS, ${ }^{\circ} 1$.

Gérard D. (1990), Rapports d'emploi et de travail, flexibilité et gestion de la main-d'œuvre : Les activités de nettoyage industriel, dans la dynamique des rapports salariaux : lieux, règles, sens, Vol. 3, in Clément et alii, Rapport pour la MIRE.

Institut de l'Entreprise (1993), «La propreté : un secteur qui contribue à l'environnement et à la qualité de la vie »,Économie et Geographie. 
Hill T.P. (1977), « On goods and services », Review of income and wealth, décembre.

Husson M. (1996), « Du ralentissement de la productivité », La Revue de l'IRES, n 22.

Michon F. (1987), «Flexibilité et marché du travail », Cahiers Français, n 231.
Pavitt K. (1984), « Sectoral Patterns of Technical Change : Towards a Taxonomy and a Theory », Research Policy, ${ }^{\circ} 13$.

Sauviat C. (1996), « Coûts de main-d'œuvre, emploi et qualité dans des marchés de services : la garde d'enfants et le nettoyage industriel », La Revue de l'IRES, $\mathrm{n}^{\circ} 22$.

\section{Le secteur du nettoyage face aux nouvelles technologies}

Par Faridah Djellal

Le secteur du nettoyage est une activité de service, initialement non informationnelle, qui connaît aujourd'hui de profondes évolutions : un élargissement et une montée en complexité de l'offre de prestations, une utilisation croissante des technologies de l'information. Ces évolutions ont des incidences importantes sur la structure des emplois de ce secteur. L'activité de nettoyage est en effet à l'origine du développement d'un certain nombre d'innovations non technologiques. Différentes trajectoires d'innovation sont mises en évidence, puis en correspondance avec la structure d'emploi de ce secteur. Pus précisément, il s'agit d'examiner les conséquences de la modification de la nature du produit de cette activité sur l'emploi, notamment sur l'émergence de nouvelles modalités de pratique de travail liées à la professionnalisation d'une partie non négligeable de cette activité. 